\title{
ULBRICH, Claudia, Shulamit und Margarete. Macht, Geschlecht und Religion in einer ländlichen Gesellschaft des 18. Jahrhunderts
}

Patrice Veit

\section{OpenEdition}

\section{Journals}

Édition électronique

URL : http://journals.openedition.org/ifha/1337

DOI : 10.4000/ifha. 1337

ISSN : 2198-8943

Éditeur

IFRA - Institut franco-allemand (sciences historiques et sociales)

Référence électronique

Patrice Veit, «ULBRICH, Claudia, Shulamit und Margarete. Macht, Geschlecht und Religion in einer ländlichen Gesellschaft des 18. Jahrhunderts », Revue de I'IFHA [En ligne], Date de recension, mis en ligne le 01 janvier 2000, consulté le 22 septembre 2020. URL : http://journals.openedition.org/ifha/1337 ; DOI : https://doi.org/10.4000/ifha.1337

Ce document a été généré automatiquement le 22 septembre 2020

(CIFHA 


\section{ULBRICH, Claudia, Shulamit und} Margarete. Macht, Geschlecht und Religion in einer ländlichen Gesellschaft des 18. Jahrhunderts

\section{Patrice Veit}

1 Cet ouvrage a pour objet une communauté rurale au XVIIIe s. forte de 700 habitants, celle de Steinbiedersdorf (aujourd'hui Pontpierre), à la frontière entre la France et l'Empire. Outre sa position frontalière, ce village offre la particularité de voir cohabiter communautés catholique et juive (un sixième de la population) et de dépendre d'une administration seigneuriale (celle des seigneurs de Créhange) protestante. Ce village pluri-confessionnel, servi par une documentation exceptionnelle, sert de cadre à C.U. pour analyser, dans une perspective d'anthropologie historique et à partir d'« histoires de vie » de femmes, les rapports complexes existant entre pouvoir, sexe et religion, et pour aborder, au-delà, le problème de l'organisation des sexes dans la société rurale à l'époque moderne et des systèmes symboliques qui s'y rapportent.

2 La première partie de l'étude est consacrée à la communauté chrétienne en retraçant la vie de quelques femmes que l'on suit dans le village, à l'église, devant la justice, dans les relations de voisinage, parcours individuels contextualisés dans les cadres socioéconomiques, politiques et culturels. La seconde partie de l'ouvrage renverse la perspective : l'histoire du village est désormais considérée du point de vue de la population juive, en retraçant l'histoire " en propre » de la communauté, cadre qui permet d'analyser ensuite quelques destins de femmes pour dégager leurs espaces propres, les réseaux et les rapports de force ainsi que la diversité et la différence des expériences féminines. Mais ce village connaît aussi des liens d'entraide entre Chrétiens et Juifs, des relations de voisinage, des réseaux et des alliances, il est également le lieu d'intérêts divergents et de conflits, ce que C.U. reconstruit avec précision dans un dernier chapitre. 
3 Par son objet et la perspective qui est la sienne, cet ouvrage, d'une écriture alerte, est une étude pionnière : en unissant deux directions de recherche - l'histoire des relations entre les sexes et l'histoire des communautés juives -, elle analyse les différents rapports de force à l'œuvre et pose, de manière neuve, la question de l'importance non seulement des hiérarchies et des inégalités dans la société d'ordres, mais aussi des marges de manœuvre possibles et des cadres de vie, des phénomènes d'inclusion et d'exclusion comme des relations qui s'échafaudent par delà les frontières sociales, religieuses ou culturelles. L'ouvrage de C.U. ouvre en outre de nouvelles voies concernant la "Geschlechtergeschichte ", en ce qu'il ne décrit pas seulement les femmes, mais aussi femmes et hommes dans l'éventail et la complexité de leurs relations. Il constitue enfin une contribution majeure à l'étude des communautés rurales de l'époque moderne (rejoignant, avec des accents néanmoins différents, les travaux essentiels de David Sabean, Hans Medick et Jürgen Schlumbohm) et apporte une réflexion particulièrement enrichissante sur les différents aspects de la notion de frontière (géographiques, sociaux, religieux, anthropologiques). Le caractère pluridimensionnel de l'étude, l'approche micro-historique et la richesse des perspectives développées rendent compte de la complexité d'un monde dont l'organisation est fondée sur la pluralité.

4 Autre atout de cette étude : une introduction fine, nuancée et particulièrement stimulante qui permettra au lecteur de mieux connaître les recherches actuelles concernant l'histoire des relations entre les sexes notamment dans le monde germanique et anglo-saxon ou les discussions critiques autour du concept de ganzes Haus, un concept hérité de Riehl et largement utilisé en Allemagne pour la période moderne sous l'influence d'Otto Brunner. 